\title{
Une nouvelle société de discipline?
}

\section{Carlos Beat Quinto}

Dr méd., membre du Comité central de la FMH, responsable du département Santé publique et professions de la santé

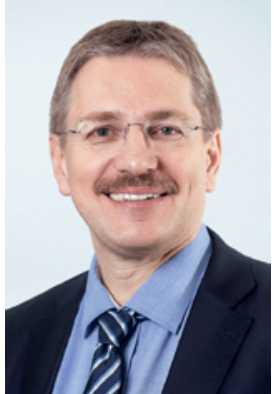

La Société suisse des médecins spécialistes en prévention et santé publique uniformise son nom dans tout le pays: en allemand, le terme de «Public Health» remplace désormais celui de "Gesundheitswesen». Vous découvrirez dans ce numéro ce que recouvre le champ d'activités de ces spécialistes. La santé et la maladie doivent toujours être envisagées du point de vue de l'individu et de la population. Le changement de perspective est souvent porteur de conflits et requiert à la fois des connaissances en médecine clinique axée sur l'individu et une formation en santé publique. De même, il convient de toujours combiner la prévention comportementale et la prévention structurelle toute autre approche étant ni cohérente ni efficace. Il existe de nombreux points communs entre les soins primaires et la santé publique. Quels problèmes faut-il résoudre? Il y a encore beaucoup à faire en Suisse, en particulier dans le domaine de la prévention structurelle de l'alcoolisme et du tabagisme. Plusieurs milliards de francs pourraient être économisés au niveau des coûts de la santé et des paiements aux caisses-maladie. Plus de 50 pays européens sont plus avancés à cet égard. Pour l'instant, notre Parlement veut encore se permettre politiquement de dépenser chaque année des milliards dans le système de santé alors qu'il serait possible de réaliser des économies en prenant de simples mesures de prévention structurelle, sans qu'aucun patient ne soit privé d'une

Les soins primaires et la santé publique ont beaucoup de points communs: il s'agit d'éviter une déshumanisation de la médecine.

prestation quelconque. Si nous souhaitons conserver notre crédibilité et notre intégrité aux yeux de la population, nous n'avons besoin d'aucun budget global ni d'aucun rationnement, mais d'objectifs dans le domaine de la prévention structurelle. En ce qui concerne le problème des coûts, notons que depuis une dizaine d'années ce sont les primes de l'assurance-maladie et non les coûts de la santé qui explosent, si l'on se réfère aux chiffres de l'office fédéral de la statistique
(MOKKE). La hausse des primes tellement facile à utiliser comme moyen de pression politique est bien plus forte que l'augmentation réelle des coûts de la santé. La Société suisse des médecins spécialistes en prévention et santé publique s'occupe également de l'interface entre sciences sociales et médecine, le thème auquel la Conférence suisse de santé publique était dédiée cette année. Il faut faire de l'advocacy. L'apprentissage de ce rôle et les compétences requises font partie intégrante du nouveau catalogue des objectifs de formation des facultés suisses de médecine (PROFILES). Les hypothèses économiques contrefaites, emprun-

\section{Le point essentiel de la prestation médicale} qui consiste à comprendre la personne malade se mesure difficilement en chiffres.

tées sans réfléchir aux processus de production et de gestion, sont à proscrire, car elles génèrent des incitatifs négatifs, menacent les valeurs éthiques de la médecine et nuisent à la motivation intrinsèque de l'ensemble des professionnels de la santé. Le maîtremot, c'est le dialogue. Les stratégies ne doivent pas être élaborées pour le corps médical, mais viser une collaboration adéquate avec le corps médical. Faute de quoi le fossé creusé par la croissance démographique et une pénurie de personnel qualifié continuera de s'approfondir. La surenchère administrative issue de la culture de la défiance ne tient pas ses promesses, à savoir la sécurité, et produit aujourd'hui déjà de graves effets secondaires. Pour Giovanni Maio, il s'agit d'éviter une déshumanisation de la médecine. Une approche commune et interprofessionnelle permettrait d'y parvenir. Les patientes et les patients ne sont pas des marchandises. Selon ce médecin, il est difficile de mesurer le point essentiel de la prestation médicale, qui consiste à comprendre les malades. Dans cet esprit, le département Santé publique et professions de la santé de la FMH souhaite poursuivre sa bonne collaboration avec la Société suisse des médecins spécialistes en prévention et santé publique. 